\title{
Tunneling spectroscopy using a probe qubit
}

\author{
A. J. Berkley ㄱ A. J. Przybysz, T. Lanting, R. Harris, N. Dickson $\}$ F. Altomare, M. \\ H. Amin, P. Bunyk, C. Enderud, E. Hoskinson, M. W. Johnson, E. Ladizinsky, R. \\ Neufeld, C. Rich, A. Yu. Smirnov, E. Tolkacheva, S. Uchaikin, and A. B. Wilson \\ D-Wave Systems Inc. 100-4401 Still Creek Dr., Burnaby BC Canada V5C 6G9
}

\begin{abstract}
We describe a quantum tunneling spectroscopy technique that requires only low bandwidth control. The method involves coupling a probe qubit to the system under study to create a localized probe state. The energy of the probe state is then scanned with respect to the unperturbed energy levels of the probed system. Incoherent tunneling transitions that flip the state of the probe qubit occur when the energy bias of the probe is close to an eigenenergy of the probed system. Monitoring these transitions allows the reconstruction of the probed system eigenspectrum. We demonstrate this method on an rf SQUID flux qubit.
\end{abstract}

PACS numbers: 85.25.Am, 85.25.Cp, 03.67.Lx, 03.65.Xp

Keywords: spectroscopy; tunneling spectroscopy; qubit; SQUID; rf SQUID; flux qubit; quantum annealing; adiabatic quantum computing; quantum computing; Ising spin

Recent technological advances have allowed the construction of mesoscale systems of individual quantum elements, including hundreds of trapped ions 1 , 14 entangled ions ${ }^{2}$, nanomagnetic systems assembled out of magnetic atoms on metallic surfaces ${ }^{3}$, ultracold ${ }^{87} \mathrm{Rb}$ atoms in optical lattices $\stackrel{4}{\underline{4}}$, and arrays of superconducting devices ${ }^{5.6}$. While the study of small numbers of atoms or devices often involves direct manipulation and full state tomography, these techniques become impractical in the mesoscale regime. As a result, there is a need for tools that are applicable when one has a mesoscale system with limited control over its individual elements.

Tunneling spectroscopy is a powerful tool for studying condensed matter systems. It has been used to push the limits of our understanding of many-body physics, as in recent studies of two dimensional electron systems in high magnetic field using time domain capacitance spectroscopy ${ }^{\underline{7}}$ and scanning tunneling spectroscopy ${ }^{8}$. Tunneling spectroscopy can also be used to directly validate numerical or analytical models of complex systems, such as the single particle states of CdSe quantum $\operatorname{dots}^{9}$ or the electronic wavefunctions of carbon nanotubes $\frac{10}{}$. Motivated by the ability of tunneling spectroscopy to probe the quantum behaviour of mesoscale systems, we have developed an analogous method that is applicable when one has limited control over a large system. The large system is probed using a dedicated probe qubit with its own readout and low bandwidth control of its Hamiltonian. We have termed this new technique qubit tunneling spectroscopy (QTS).

A related method has been proposed in Ref. 11 where the probe qubit must be perturbatively coupled to the system under study. In QTS, the requirement for this weak coupling has been removed through the use of a compensation bias (as explained below). Further, the algorithm of Ref. 11 is designed to operate on a gate model quantum computer while we demonstrate QTS on a system with much more limited control.

QTS requires a probe qubit $P$ that can be described by a generic two-level system Hamiltonian:

$$
\hat{H}_{P}=-\frac{1}{2} \Delta_{P} \hat{\sigma}_{x, P}-\frac{1}{2} \epsilon_{P} \hat{\sigma}_{z, P}
$$

where $\hat{\sigma}_{x, P}$ and $\hat{\sigma}_{z, P}$ are Pauli matrices operating on $P$, and both parameters $\epsilon_{P}$ and $\Delta_{P}$ should be controllable. The eigenstates of $\hat{\sigma}_{z, P}$ with eigenvalues +1 and -1 are $|\uparrow\rangle_{P}$ and $|\downarrow\rangle_{P}$, respectively. The $\hat{\sigma}_{z, P}$ eigenstates should be distinguishable by a readout mechanism. Let there be a system $S$, governed by some Hamiltonian $\hat{H}_{S}$, that one would like to study.

To perform QTS we require a coupling between the probe qubit and a parameter of the system (described by an operator $\hat{C}$ ) as well as a controllable compensation bias $\epsilon_{\text {comp }}$ coupled to that same parameter. In this case, the system plus probe Hamiltonian can be expressed as:

$$
\hat{H}_{S+P}=\hat{H}_{S}+\hat{H}_{P}+J \hat{\sigma}_{z, P} \hat{C}+\frac{1}{2} \epsilon_{\mathrm{comp}} \hat{C},
$$

with $J$ the strength of the probe qubit-system interaction.

For general $\epsilon_{\text {comp }}$ the eigenstates of $S+P$ are not representative of those of $S$. However, in the special case $\epsilon_{\text {comp }}=-2 J$, the spectrum of $\hat{H}_{S+P}$ splits into two qualitatively different manifolds, $\mathcal{M}_{\uparrow}$ and $\mathcal{M}_{\downarrow}$, wherein the probe qubit $P$ is in state $|\uparrow\rangle_{P}$ and $|\downarrow\rangle_{P}$, respectively. $H_{S+P}$ can then be rewritten as:

$$
\begin{aligned}
\hat{H}_{S+P}= & \left(\epsilon_{P} \hat{\mathcal{I}}_{S}-2 J \hat{C}+\hat{H}_{S}\right) \otimes|\downarrow\rangle_{P}\left\langle\left.\downarrow\right|_{P}\right. \\
& +\hat{H}_{S} \otimes|\uparrow\rangle_{P}\left\langle\left.\uparrow\right|_{P}\right. \\
- & \frac{\Delta_{P}}{2} \hat{\mathcal{I}}_{S} \otimes\left(|\downarrow\rangle_{P}\left\langle\left.\uparrow\right|_{P}+\mid \uparrow\right\rangle_{P}\left\langle\left.\downarrow\right|_{P}\right)\right.
\end{aligned}
$$

where $\hat{\mathcal{I}}_{S}$ is the identity operator on system $S$. In the case where the third line of Eqn. 3is perturbatively small, the first line is the Hamiltonian of $\mathcal{M}_{\downarrow}$ and the second line that of $\mathcal{M}_{\uparrow}$. Thus, the energy spectrum of $\mathcal{M}_{\uparrow}$ is identical to that of $\hat{H}_{S}$. Further, the first term of the first line of Eqn. 3 shows that the energy of all states 


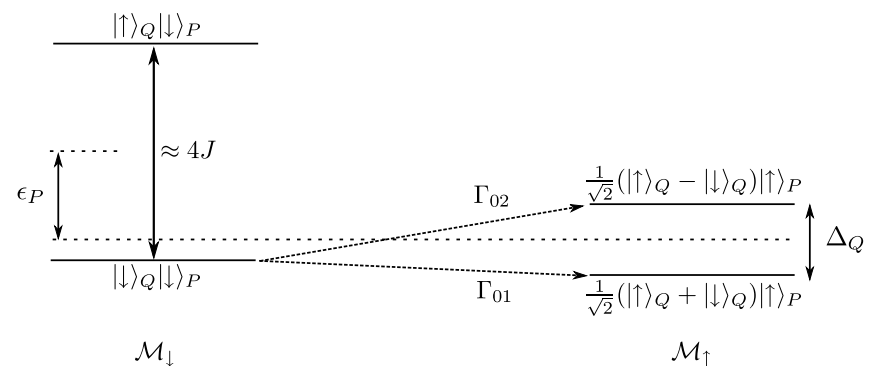

FIG. 1. Energy level diagram for the $Q+P$ qubit system described by the Hamiltonian in Eqn. 4at $\epsilon_{Q}=0$ and $\epsilon_{\mathrm{comp}}=$ $-2 J$. The system separates into two distinct manifolds, $\mathcal{M}_{\downarrow}$ and $\mathcal{M}_{\uparrow}$, that differ in the orientation of the probe qubit $P$. Spinor notation indicates state of qubits $Q$ and $P$ on the left and right, respectively. Allowing for weak tunneling in qubit $P$ facilitates incoherent tunneling processes $\Gamma_{01}$ and $\Gamma_{02}$ between the manifolds. QTS proceeds by initializing the system in the lowest energy state of $\mathcal{M}_{\downarrow}$ and finding values of $\epsilon_{P}$ for which transition rate $\Gamma$ peaks due to resonant processes $\Gamma_{01}$ and $\Gamma_{02}$.

in $\mathcal{M}_{\downarrow}$ can be shifted with respect to those of $\mathcal{M}_{\uparrow}$ by adjusting the probe energy bias $\epsilon_{P}$. For small enough $\Delta_{P}$, the third line of Eqn. 3 gives rise to incoherent intermanifold tunneling $\frac{12}{2}$ between any state $\left|k_{\downarrow}^{\prime}\right\rangle|\downarrow\rangle_{P}$ of $\mathcal{M}_{\downarrow}$ and any state $|k\rangle|\uparrow\rangle_{P}$ of $\mathcal{M}_{\uparrow}$ with a rate proportional to $\left|\Delta_{P}\left\langle k_{\downarrow}^{\prime} \mid k\right\rangle\right|^{2}$.

The QTS method begins by initializing the system into the lowest energy state of $\mathcal{M}_{\downarrow}$. The tunneling rate between manifolds peaks when an eigenstate of $\mathcal{M}_{\uparrow}$ is brought into resonance with the initial system state in $\mathcal{M}_{\downarrow}$ by adjusting $\epsilon_{P}$. This resonant tunneling transition between manifolds flips the state of the probe qubit, which can be easily detected. Thus, to perform QTS, one measures the initial transition rate $\Gamma \equiv|d P / d t|_{t=0}$, where $P$ is the probability of observing the probe qubit in its initial state, as a function of $\epsilon_{P}$, the probe energy bias. Scanning $\epsilon_{P}$ and locating peaks in $\Gamma$ allows one to map out the eigenspectrum of $\mathcal{M}_{\uparrow}$ which is identical to that of $\hat{H}_{S}$ if the compensation bias $\epsilon_{\mathrm{comp}}$ is set to $-2 J$. Note that errors in this compensation bias will skew the energy spectrum of the probed system. The errors in the extracted energy spacings of the spectrum are bounded by the compensation bias error.

To experimentally demonstrate QTS we take a target system comprising a single qubit $Q$, governed by a Hamiltonian $\hat{H}_{Q}$ (Eqn. 1 with $P \rightarrow Q$ ). We couple the probe qubit $P$ with strength $J$ through a mutual $\hat{\sigma}_{z}$ interaction to qubit $Q$. In this case, $\hat{H}_{S} \rightarrow \hat{H}_{Q}$ and $\hat{C} \rightarrow \hat{\sigma}_{z, Q}$ in Eqn. 2, yielding

$$
\hat{H}_{Q+P}=\hat{H}_{Q}+\hat{H}_{P}+J \hat{\sigma}_{z, P} \hat{\sigma}_{z, Q}+\frac{1}{2} \epsilon_{c o m p} \hat{\sigma}_{z, Q} .
$$

$\Delta_{P}$ is chosen to be small compared with all other terms so the eigenstates of the probe are to good approximation $|\uparrow\rangle_{P}$ and $|\downarrow\rangle_{P}$. Setting $\epsilon_{\text {comp }}=-2 J$ then yields a Hamiltonian of the form given in Eqn. 3. For the particular case
$\epsilon_{Q}=0$, we show how the theoretical energy spectrum of the coupled two qubit system splits into two manifolds in Fig. 1. Note that the eigenstates of $\mathcal{M}_{\uparrow}$ shown therein are superpositions of the $|\uparrow\rangle_{Q}$ and $|\downarrow\rangle_{Q}$ states. Changing $\epsilon_{P}$ allows the lowest state in $\mathcal{M}_{\downarrow},|\downarrow\rangle_{Q}|\downarrow\rangle_{P}$, to be brought into resonance with the states in $\mathcal{M}_{\uparrow}$. The system plus probe can tunnel from $\mathcal{M}_{\downarrow}$ to $\mathcal{M}_{\uparrow}$ through the incoherent processes labelled as $\Gamma_{01}$ and $\Gamma_{02}$.

The two qubits, probe $P$ and target $Q$, used in the experiment were rf SQUID flux qubits on a D-Wave quantum annealing processor thermalized to a temperature $T=12 \mathrm{mK}$. A description of a chip similar to that used in this study can be found in Ref. 13. The low energy rf SQUID flux qubit Hamiltonian ${ }^{14}$ has a direct mapping onto Eqn. 1.

$$
\hat{H}_{Q}=-\frac{1}{2} \Delta_{Q}\left(\Phi_{Q}^{c c j j}\right) \hat{\sigma}_{x, Q}-\Phi_{Q}^{x}\left|I_{Q}^{p}\left(\Phi_{Q}^{c c j j}\right)\right| \hat{\sigma}_{z, Q}
$$

where we have performed the substitution $\Delta_{Q} \rightarrow$ $\Delta_{Q}\left(\Phi_{Q}^{c c j j}\right)$ and $\epsilon_{Q} \rightarrow 2\left|I_{Q}^{p}\left(\Phi_{Q}^{c c j j}\right)\right| \Phi_{Q}^{x}$, with $\Phi_{Q}^{c c j j}$ and $\Phi_{Q}^{x}$ being externally controlled flux biases and $\left|I_{Q}^{p}\left(\Phi_{Q}^{c c j j}\right)\right|$ being the magnitude of the qubit persistent current. Note that both $\Delta_{Q}$ and $\epsilon_{Q}$ (through $\left|I_{Q}^{p}\right|$ ) are functions of $\Phi_{Q}^{c c j j}$. The functional forms of these dependencies are determined by the physical parameters of the rf SQUID, as described in detail in Ref. 14. If one considers the qubit $Q$ as an Ising spin, then $\Delta_{Q}$ corresponds to a transverse magnetic field, $I_{Q}^{p}$ is the magnitude of the spin, and $\Phi_{Q}^{x}$ is an applied longitudinal magnetic field. The physical Hamiltonian for the probe qubit $\hat{H}_{P}$ is found by replacing $Q$ by $P$ in Eqn. 5. The probe qubit had a persistent current $\left|I_{P}^{p}\right|=1.0 \mu \mathrm{A}$ and $\Delta_{P} / h \sim 1 \mathrm{MHz}$. The small $\Delta_{P}$ was chosen so that the transition rate $\Gamma$ of the probe qubit was contained within the dc to $3 \mathrm{MHz}$ bandwidth of our slow control lines and to satisfy the incoherent inter-manifold tunneling condition. An on-chip tunable coupler ${ }^{13}$ between the two qubits was programmed to attain an interqubit mutual inductance $M=2.0 \mathrm{pH}$. The resulting form for $J$ in Eqn. 团 is $J=M\left|I_{Q}^{p}\right|\left|I_{P}^{p}\right|$. With these parameters, the spectral gap in the $\mathcal{M}_{\downarrow}$ manifold, as depicted in Fig. 1, satisfied $4 J=4 M\left|I_{Q}^{p}\right|\left|I_{P}^{p}\right| \gg k_{B} T$ over the range of $\left|I_{Q}^{p}\right|$ encountered in these experiments. Consequently, there was negligible thermal activation out of the initial state $|\downarrow\rangle_{Q}|\downarrow\rangle_{P}$ to higher levels within $\mathcal{M}_{\downarrow}$. With these parameters, the compensation bias is explicitly $\frac{1}{2} \epsilon_{\text {comp }} \hat{\sigma}_{z, Q}=-J \hat{\sigma}_{z, Q}=-M\left|I_{Q}^{p}\right|\left|I_{P}^{p}\right| \hat{\sigma}_{z, Q}$ and is applied by adding an offset $M\left|I_{P}^{p}\right| \sim 1 \mathrm{~m} \Phi_{0}$ to the flux bias $\Phi_{Q}^{x}$ of qubit $Q$. This compensation bias requires only careful calibration of probe parameters.

The experimental method for initialization and readout is the same as the two-qubit cotunneling technique described in Ref. 15. The experiments described herein differed from the cotunneling experiment in three regards: First, in QTS one intentionally sets $\Delta_{P} \ll \Delta_{Q}$, thus exploring an extreme limit of the mismatched tunneling energy configuration described in Ref. 15. Second, 
(a)
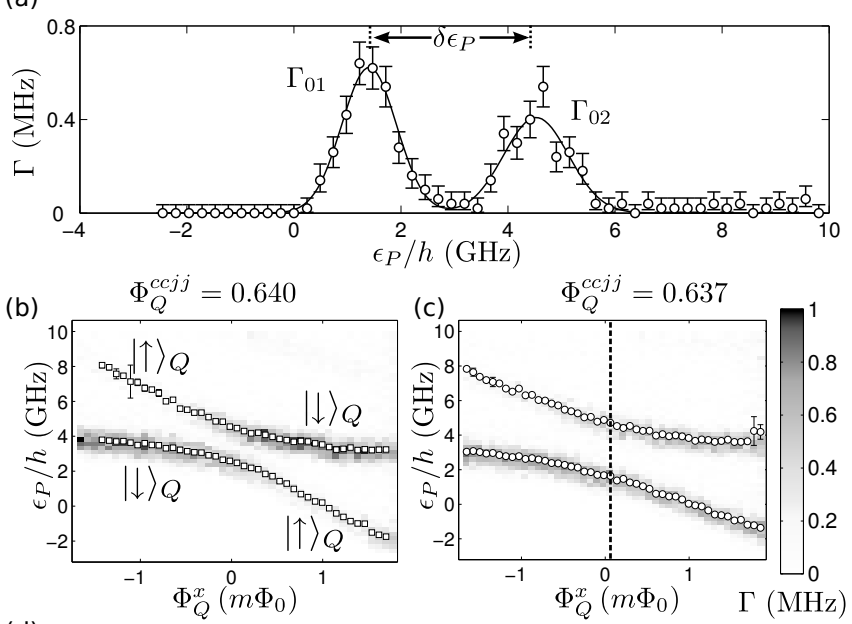

(d)

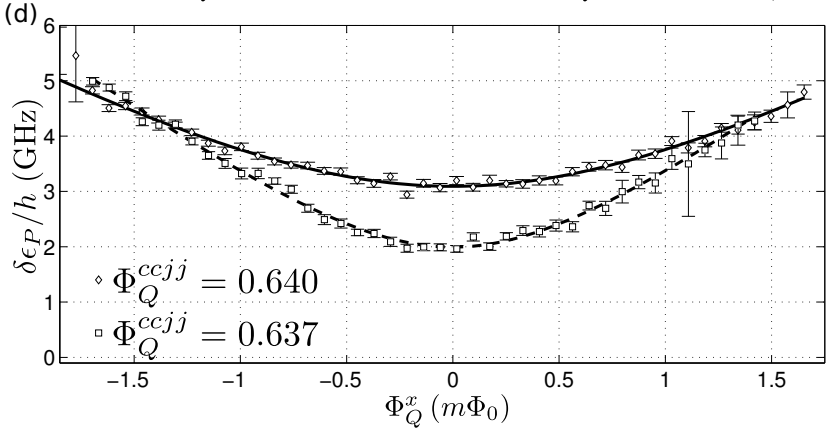

FIG. 2. (a) QTS tunneling rate data versus $\epsilon_{p}$ for $\Phi_{Q}^{x}=$ $0.1 \mathrm{~m} \Phi_{0}$ and $\Phi_{Q}^{c c j j}=0.637$. Peaks in $\Gamma$ are readily associated with the processes $\Gamma_{01}$ and $\Gamma_{02}$ denoted in Fig. 1 The centers of the peaks are found by fitting to a sum of two Gaussian peaks. (b) Qubit $Q$ energy spectra obtained by QTS at control bias values: $\Phi_{Q}^{c c j j} / \Phi_{0}=0.640$; (c) $\Phi_{Q}^{c c j j} / \Phi_{0}=0.637$. In both plots, the ordinate is the probe bias energy $\epsilon_{P}=2\left|I_{P}^{p}\right| \Phi_{P}^{x}$ and the abscissa is the flux bias $\Phi_{Q}^{x} \propto \epsilon_{Q}$ applied to qubit $Q$. The grayscale indicates the transition rate $\Gamma$ in the $\left(\Phi_{Q}^{x}, \epsilon_{P}\right)$ plane. White circles denote the centers of peaks in $\Gamma$ found by the Gaussian fits. Avoided crossings between two localized states, explicitly labelled in (b) as $|\uparrow\rangle_{Q}$ and $|\downarrow\rangle_{Q}$, are visible. (d) The difference in probe energy $\delta \epsilon_{P}$ between the two peak centers as a function of $\Phi_{q}^{x}$ for the datasets in (a) and (b). Results have been fit to the dispersion of the Hamiltonian in Eqn. [5 using $\Delta_{Q}$ and $\left|I_{Q}^{p}\right|$ as free parameters.

in QTS we use relatively large offset biases $\epsilon_{\text {comp }} /\left|I_{Q}^{p}\right|=$ $1 \mathrm{~m} \Phi_{0}$ in order to satisfy the compensation condition embodied in Eqn. 3. Third, whereas the dynamics studied in Ref. 15 involved incoherent tunneling of the pair of qubits between localized initial and final spin states, in QTS the final state can place qubit $Q$ in a delocalized (superposition) state, as depicted in Fig. 1,

A scan of the initial transition rate $\Gamma$ versus $\epsilon_{P}$ at $\Phi_{Q}^{x} \sim 0$ and $\Phi_{Q}^{c c j j}=0.637$ is shown in Fig. 2(a). The data clearly show two distinct peaks. These peaks are readily identified as the processes $\Gamma_{01}$ and $\Gamma_{02}$ indicated in Fig. 1. We fit such scans to a model composed of a

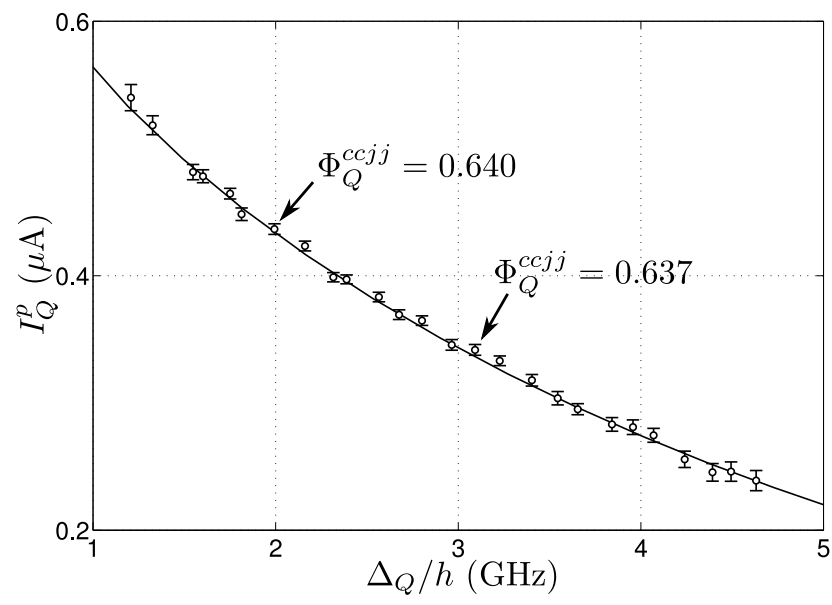

FIG. 3. $I_{Q}^{p}\left(\Phi_{Q}^{c c j j}\right)$ vs $\Delta_{Q}\left(\Phi_{Q}^{c c j j}\right)$ from the $Q$ spectra for a range of settings of the control bias $\Phi_{Q}^{c c j j}$. The data points from the fits in Fig. 2 (d) are labelled with arrows. The data also have horizontal error bars approximately the size of the symbols. Results have been fit to the rf SQUID model from Ref. 14 (solid line) with the rf SQUID capacitance $C$ as the only free parameter.

pair of Gaussian peaks in order to locate the peak centers. Example maps of the initial transition rate $\Gamma$ versus $\epsilon_{P}$ for a range of qubit $Q$ flux biases around $\Phi_{Q}^{x}=0$ are shown in Figs. 2(b) and (c) for two values of the control flux bias $\Phi_{Q}^{c c j j}$ (and therefore $\Delta_{Q}$ ). For clarity the centers of the Gaussian peaks have been indicated by white circles. The peak positions reveal the avoided crossing between localized spin states $|\uparrow\rangle_{Q}$ and $|\downarrow\rangle_{Q}$. In Fig. 2(d), we summarize the difference in probe energy $\delta \epsilon_{P}$ between the two peaks as a function of $\Phi_{Q}^{x}$. We then fit those results to the eigenspectrum of the Hamiltonian in Eqn. 5. using $\Delta_{Q}$ and $\left|I_{Q}^{p}\right|$ as free parameters.

In order to crosscheck our QTS results, we have repeated the measurements and analysis that led to Fig. 2(d) for several values of $\Phi_{Q}^{c c j j}$. By doing so, we generated maps of the qubit parameters $\Delta_{Q}$ and $\left|I_{Q}^{p}\right|$ as a function of $\Phi_{Q}^{c c j j}$. A plot of the relationship between $\left|I_{Q}^{p}\right|$ and $\Delta_{Q}$ is shown in Fig. 3. This curve is completely determined by the rf SQUID inductance $L$ and capacitance $C$ of qubit Q. We have fit these results (solid line in Fig. 3) to a physical rf SQUID Hamiltonian (Eqn. 4 in Ref. 14) taking $L=355.5 \mathrm{pH}$, as determined by independent measurements, and using $C$ as a free parameter. The best fit returned $C=118 \pm 2 \mathrm{fF}$, which is a physically reasonable value, given Josephson junction sizes and qubit wiring geometry. The single parameter fit models the data well, implying that QTS has correctly extracted the low energy spectrum of $\operatorname{rf}$ SQUID qubit $Q$.

QTS could, in principle, yield more than just the eigenspectrum of system $S$. In particular, there is significant information contained in the spectral weight of the peaks in $\Gamma$. For example, the spectral lines inferred 
from Fig. 2(b) are less pronounced for the upper level at $\Phi_{Q}^{x}<0$ and for the lower level at $\Phi_{Q}^{x}>0$. This is due to the proportionality of the initial transition rate $\Gamma$ to the small overlap of the initial state of $Q\left(\sim|\downarrow\rangle_{Q}\right)$ with its final state $\left(\sim|\uparrow\rangle_{Q}\right)$. Choosing $\epsilon_{\text {comp }}=+2 J$, instead of $-2 J$, yields a system in which the states in $\mathcal{M}_{\downarrow}$ exchange roles, thus yielding a new initial state $|\uparrow\rangle_{Q}|\downarrow\rangle_{P}$. Repeating the QTS experiment with this configuration should then swap the regions of high and low peak visibility seen in Fig. 2(b). Thus the spectral weight contains information about the wavefunction of the probed system.

Further information could be gleaned from the lineshapes of inter-manifold tunneling processes. We chose to fit tunneling rate peaks to Gaussians as we had anticipated that their lineshapes would be dominated by the incoherent tunneling of the slow probe qubit $P$, as in Ref. 16. A detailed analysis of the physical mechanisms that lead to particular lineshapes is currently underway.

We have demonstrated a low bandwidth method, termed qubit tunneling spectroscopy (QTS), by probing the energy spectrum of a first qubit by using a second probe qubit to split the two qubit system into two man- ifolds of qualitatively different states. Transitions between the two manifolds are monitored as a function of the energy bias of one of the manifolds. Transition rate peaks correspond to the presence of eigenstates in the target manifold under study at that energy bias. We validated this method by verifying that rf SQUID flux qubit energy spectra measured in this manner are consistent with an rf SQUID Hamiltonian. QTS provided a direct measurement of the first qubit's energy splitting $\Delta_{Q}$ that was three orders of magnitude larger than the measurement bandwidth $\Delta_{P}$.

While the demonstration in this paper was limited to a single qubit, QTS is extensible to larger numbers of qubits and to other physical systems, provided one has good control and readout of the probe and a method of applying a compensation bias. We anticipate that QTS will be a valuable tool for studying mesoscale systems.

The authors would like to acknowledge: F. Cioata, P. Spear for the design and maintenance of electronics control systems; D. Bruce, P. deBuen, M. Gullen, M. Hager, G. Lamont, L. Paulson, C. Petroff, A. Tcaciuc for cryogenics and IO support; I. Perminov for software design and support. *ajb@dwavesys.com

$\dagger$ Current address: Side Effects Software Inc. 1401-123 Front St. West, Toronto ON Canada M5J 2M2

1 J. W. Britton, B. C. Sawyer, A. C. Keith, C. C. J. Wang, J. K. Freericks, H. Uys, M. J. Biercuk, and J. J. Bollinger, Nature 484, 489 (Apr 2012), ISSN 0028-0836, http://dx.doi.org/10.1038/nature10981

2 T. Monz, P. Schindler, J. T. Barreiro, M. Chwalla, D. Nigg, W. A. Coish, M. Harlander, W. Hänsel, M. Hennrich, and R. Blatt, Phys. Rev. Lett. 106, 130506 (Mar 2011), http://link.aps.org/doi/10.1103/PhysRevLett.106.130506

3 A. A. Khajetoorians, J. Wiebe, B. Chilian, S. Lounis, S. Blugel, and R. Wiesendanger, Nat Phys 8, 497 (Jun 2012), ISSN 1745-2473, http://dx.doi.org/10.1038/nphys2299

4 C. Weitenberg, M. Endres, J. F. Sherson, M. Cheneau, P. Schausz, T. Fukuhara, I. Bloch, and S. Kuhr, Nature 471, 319 (Mar 2011), ISSN 0028-0836, http://dx.doi.org/10.1038/nature09827

5 M. W. Johnson, M. H. S. Amin, S. Gildert, T. Lanting, F. Hamze, N. Dickson, R. Harris, A. J. Berkley, J. Johansson, P. Bunyk, E. M. Chapple, C. Enderud, J. P. Hilton, K. Karimi, E. Ladizinsky, N. Ladizinsky, T. Oh, I. Perminov, C. Rich, M. C. Thom, E. Tolkacheva, C. J. S. Truncik, S. Uchaikin, J. Wang, B. Wilson, and G. Rose, Nature 473, 194 (May 2011), ISSN 0028-0836, http://dx.doi.org/10.1038/nature10012

6 M. Mariantoni, H. Wang, T. Yamamoto, M. Neeley, R. C. Bialczak, Y. Chen, M. Lenander, E. Lucero, A. D. OConnell, D. Sank, M. Weides, J. Wenner, Y. Yin, J. Zhao, A. N. Korotkov, A. N. Cleland, and J. M. Martinis, Science 334, 61 (2011), http://www.sciencemag.org/content/334/6052/61. abstract

7 O. E. Dial, R. C. Ashoori, L. N. Pfeiffer, and K. W.
West, Nature 464, 566 (Mar 2010), ISSN 0028-0836, http://dx.doi.org/10.1038/nature08941

8 Y. J. Song, A. F. Otte, Y. Kuk, Y. Hu, D. B. Torrance, P. N. First, W. A. de Heer, H. Min, S. Adam, M. D. Stiles, A. H. MacDonald, and J. A. Stroscio, Nature 467, 185 (Sep 2010), ISSN 0028-0836, http://dx.doi.org/10.1038/nature09330

9 E. P. A. M. Bakkers, Z. Hens, A. Zunger, A. Franceschetti, L. P. Kouwenhoven, L. Gurevich, and D. Vanmaekelbergh, Nano Letters 1, 551 (2001), http://pubs.acs.org/doi/abs/10.1021/nl015572b

10 S. G. Lemay, J. W. Janssen, M. van den Hout, M. Mooij, M. J. Bronikowski, P. A. Willis, R. E. Smalley, L. P. Kouwenhoven, and C. Dekker, Nature 412, 617 (Aug 2001), ISSN 0028-0836, http://dx.doi.org/10.1038/35088013

11 H. Wang, S. Ashhab, and F. Nori, Phys. Rev. A 85, $062304 \quad$ (Jun 2012), http://link.aps.org/doi/10.1103/PhysRevA.85.062304

12 M. H. S. Amin and D. V. Averin, Phys. Rev. Lett. 100, 197001 (May 2008), http://link.aps.org/doi/10.1103/PhysRevLett.100.197001

13 R. Harris, M. W. Johnson, T. Lanting, A. J. Berkley, J. Johansson, P. Bunyk, E. Tolkacheva, E. Ladizinsky, N. Ladizinsky, T. Oh, F. Cioata, I. Perminov, P. Spear, C. Enderud, C. Rich, S. Uchaikin, M. C. Thom, E. M. Chapple, J. Wang, B. Wilson, M. H. S. Amin, N. Dickson, K. Karimi, B. Macready, C. J. S. Truncik, and G. Rose, Phys. Rev. B 82, 024511 (Jul 2010), http://link.aps.org/doi/10.1103/PhysRevB.82.024511

14 R. Harris, J. Johansson, A. J. Berkley, M. W. Johnson, T. Lanting, S. Han, P. Bunyk, E. Ladizinsky, T. Oh, I. Perminov, E. Tolkacheva, S. Uchaikin, E. M. Chapple, C. Enderud, C. Rich, M. Thom, J. Wang, B. Wil- 
son, and G. Rose, Phys. Rev. B 81, 134510 (Apr 2010), http://link.aps.org/doi/10.1103/PhysRevB.81.134510

15 T. Lanting, R. Harris, J. Johansson, M. H. S. Amin, A. J. Berkley, S. Gildert, M. W. Johnson, P. Bunyk, E. Tolkacheva, E. Ladizinsky, N. Ladizinsky, T. Oh, I. Perminov, E. M. Chapple, C. Enderud, C. Rich, B. Wilson, M. C. Thom, S. Uchaikin, and G. Rose, Phys. Rev. B 82, 060512 (Aug 2010),
http://link.aps.org/doi/10.1103/PhysRevB.82.060512

16 R. Harris, M. W. Johnson, S. Han, A. J. Berkley, J. Johansson, P. Bunyk, E. Ladizinsky, S. Govorkov, M. C. Thom, S. Uchaikin, B. Bumble, A. Fung, A. Kaul, A. Kleinsasser, M. H. S. Amin, and D. V. Averin, Phys. Rev. Lett. 101, 117003 (Sep 2008), http://link.aps.org/doi/10.1103/PhysRevLett.101.117003 